\title{
MANEJO COMUNAL DE FAUNA SILVESTRE EN EL PARQUE NACIONAL CORDILLERAAZUL, SAN MARTÍN - PERÚ
}

Jorge Watanabe Sato ${ }^{1}$

\begin{abstract}
RESUMEN
Situado entre los ríos Huallaga y Ucayali, el Parque Nacional Cordillera Azul (PNCAZ), fue establecido el 2001, abarcando más de 1,3 millones de hectáreas. Para iniciar el desarrollo del programa y sus actividades, se realizaron previamente revisiones de información, como la obtenida del Mapeo de Usos y Fortalezas (MUF), de investigaciones de usos de recursos desarrolladas en la zona de amortiguamiento del parque y de la realidad local de las comunidades involucradas. En este primer año de manejo, el programa se desarrolla con la participación de dos sectores, estos son Shamboyacu y Pikiyacu, además se tuvieron avances importantes en los sectores de Chazuta, Cushabatay y en menor medida en el sector de Pucayacu. La metodología del programa de manejo comunal de fauna presentó los siguientes pasos: 1) evaluación, 2) recopilación y análisis de información básica, 3) diseño, 4) identificación de usuarios y de áreas de uso, 5) elaboración de las normas de uso, 6) elaboración de la propuesta del manejo comunal, 7) implementación de las actividades de manejo, 8) monitoreo del proceso y, 9) análisis de los resultados.

Se espera que el parque y la zona de amortiguamiento podrían ser conocidos por la gente local como un sistema de fuente-sumidero, con el PNCAZ proporcionando fuentes de poblaciones de fauna que podrían ser aprovechadas dentro de la zona de amortiguamiento (sumidero).
\end{abstract}

Palabras claves: zona de amortiguamiento, manejo comunal, sostenibilidad.

\section{SUMMARY}

Located between the rivers Huallaga and Ucayali, the National Park Blue Mountain range, was established the 2001, including more than 1.3 million hectares. In order to initiate the development of the program and its activities, revisions of information were made previously, like the obtained one from the Mapeo de Usos and Fortalezas (MUF), of investigations uses of resources developed in the zone of damping of the park and the local reality of the involved communities. In this first year of handling, the program is developed with the participation of two sectors, these are Shamboyacu and Pikiyacu, in addition important advances in the sectors of Chazuta, Cushabatay and to a lesser extent in the sector of Pucayacu were had. The process of the handling program presented displayed the following steps: 1) evaluation, 2) compilation and basic analysis of information, 3) design, 4) identification of users and areas of use, 5) elaboration of the use norms, 6) elaboration of the proposal of the communal handling, 7) implementation of the handling activities, 8) monitored of the process and, 9) analysis of the results. One hopes that the park and the zone of damping could be known by agricultura, sino también por actividades de caza, pesca y extracción maderera como una manera de subsistencia.

Key Words: zone of damping, commural handling, sostenibility.

\footnotetext{
${ }^{1}$ Centro de Conservación, Investigación y Manejo de Áreas (CIMA)

E-mail:jwatanabesato@hotmail.com Jr. Huiracocha 1775, Jesús María - Lima, Perú
} 


\section{INTRODUCCIÓN}

Como parte de la información de diagnóstico socio-económico para la elaboración del Plan Maestro del Parque Nacional Cordillera Azul, desarrollamos el mapa de uso actual de recursos, que incluye información sobre uso de fauna silvestre en las comunidades vecinas al parque, que ha servido como base para el diseño de las actividades de manejo de fauna.

La estrategia del plan de manejo comunal de fauna dentro y fuera del parque, es la participación de los pobladores locales (comunidades rurales, ribereñas y nativas) y sus particularidades socio-culturales en las propuestas de implementación y desarrollo de las actividades. De esta forma el programa se interiorizó entre los pobladores, elevando su importancia como estrategia de conservación. Se partió de una propuesta externa a las comunidades, pero evitándose en el diseño, manipulaciones, imposiciones y decisiones externas; pero por otro lado se respetó las normas y reglamentaciones vigentes, referente a las áreas naturales protegidas y la fauna silvestre (Watanabe, 2005).

La tarea de implementación gradual y participativa del plan de manejo, con miras a establecer alianzas con las comunidades, tiene como fin la recuperación progresiva de las poblaciones de fauna al interior del parque (Gavin, 2002). De esta forma, las poblaciones de fauna recuperada servirían de suministro o fuente para repoblar las áreas de uso de las comunidades en la zona de amortiguamiento, en base al modelo de «fuente-sumidero» (Novaro et al., 2000).

\section{ÁREA DE MANEJO}

Las actividades de manejo se realizan dentro de la denominadas zonas de recuperación propuestas según el Plan Maestro del PNCAZ (CIMA, 2004) y de la zona de amortiguamiento colindante con las comunidades. En este sentido el área es compatible tanto con el uso sostenible de los recursos naturales con fines de subsistencia, como con el desarrollo de actividades enmarcadas en este manejo comunal elaborado con la participación de la población local.

El programa de manejo comunal de fauna silvestre se desarrolla actualmente con la participación de las comunidades del sector Shamboyacu y Pikiyacu, ubicadas en el lado noroeste de la zona de amortiguamiento del PNCAZ. Además se viene iniciando acciones en otros sectores del parque, con la finalidad de replicar esta experiencia.

El primer sector de trabajo, agrupa a las comunidades ubicadas en el valle del Ponasa, en el distrito de Shamboyacu, provincia de Picota, departamento de San Martín, en la zona amortiguamiento del PNCAZ. El segundo sector de trabajo se encuentra ubicado en el valle del Pikiyacu, en el distrito del Alto Biavo, provincia de Bellavista, departamento de San Martín, en la zona de amortiguamiento del PNCAZ (Figura 1).

\section{METODOLOGÍA}

Las acciones iniciales para diseñar participativamente las actividades de manejo comunal de fauna silvestre, se basaron en la información del MUF (Mapeo de Usos y Fortalezas), la cual es una metodología participativa diseñada para la recolección de datos, la cual fue realizada en 53 comunidades vecinas al parque. Esta herramienta permitió (1) reunir información cualitativa y cuantitativa sobre los patrones de uso de recursos, y (2) realizar un mapeo de las áreas de caza y pesca dentro y fuera del parque (Wali et al., 2004).

Se desarrollaron talleres de Visión del Plan Maestro, donde se recogieron las expectativas de la población vecina con respecto a los servicios ambientales y bienes que brinda el parque, a fin de consolidarla en una visión general sobre sus comunidades. Para validar el interés de las comunidades, se desarrollaron los Acuerdos Azules, los cuales son compromisos de conservación entre el Parque y las comunidades vecinas, orientadas al uso compatible de los recursos y la protección del parque. 


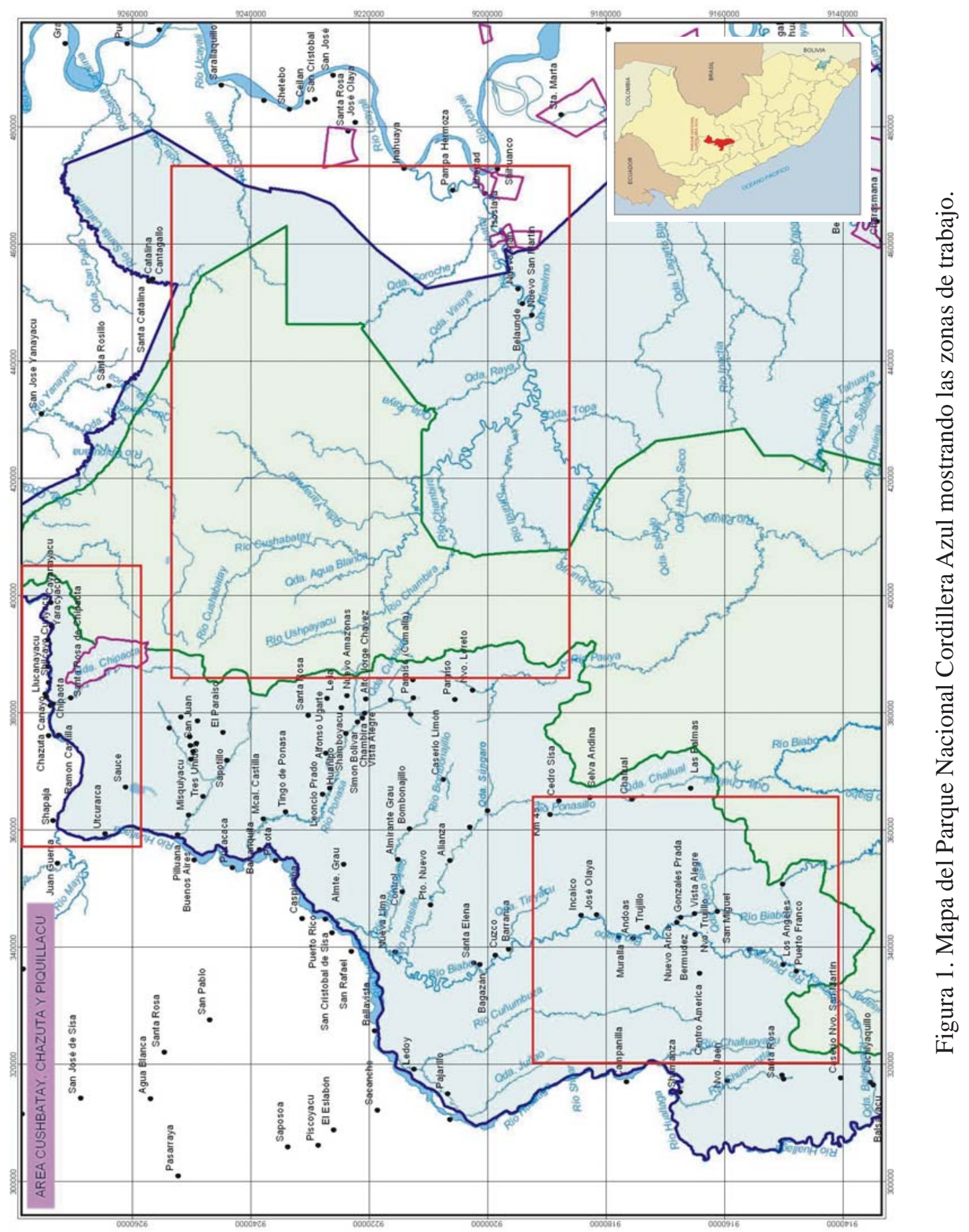


La metodología empleada en este plan de manejo comunal de fauna, presenta los siguientes pasos: 1) evaluación, 2) recopilación de información básica, 3) diseño, 4) identificación de usuarios y de áreas de uso, 5) elaboración de las normas de uso, 6) elaboración de la propuesta del manejo comunal, 7) implementación de las actividades de manejo, 8) monitoreo del proceso y, 9) análisis de los resultados.

Para calcular la cuota de extracción sustentable de animales en las áreas de uso, utilizamos el modelo de cosecha sostenible de Robinson y Redford (1991), basado en los conceptos de producción máxima, entendida como la producción (animales por kilómetro cuadrado) generada durante un año por una población silvestre bajo condiciones ambientales y extracción sustentable óptimas, alcanzada únicamente si la producción durante ese año alcanzó niveles máximos (Zapata, 2001).

\section{RESULTADOS}

En este artículo presentaremos los datos obtenidos por las comunidades del sector Shamboyacu. Estas comunidades utilizan las áreas de manejo, localizadas en la zona de recuperación del parque, a lo largo de la cuenca del Río Ushpayacu y presenta cinco áreas de uso (caza y pesca), teniendo éstas una extensión de 51 kilómetros cuadrados, las mencionadas áreas son las siguientes: Avejaico-Muquichal (5 km2), HuecoGavicho (15 km2), Satalaya-Flores (7 km2), Varadero-Aguanal (10 km2) y TenazoaHuascayacu (14 km2).

El manejo comunal de fauna silvestre está siendo desarrollado por los pobladores de las comunidades de Nuevo Amazonas, Lejía, Simón Bolívar, Chambira, Vista Alegre, Alto Jorge Chávez, Paraíso y Alto Ponasa (Watanabe, 2004).

Los pobladores locales cuentan con áreas permitidas para el uso de fauna, áreas de protección (no uso), animales permitidos, cuotas de caza, calendarios de caza y pesca, y formularios de registro. Por otro lado se han elaborado diversos materiales de información, lo cuales fueron proporcionados a los pobladores locales; entre ellos tenemos, láminas de fauna silvestre, mapas detallados de la zona, poster de las fases del trabajo, entre otros. Los registros de caza indican un estimado anual de 113 animales cazados en las áreas de uso del parque y de 226 animales cazados en al zona de amortiguamiento. Las ocho comunidades del sector involucradas en este proceso cazan con mayor frecuencia en las áreas de uso del parque, los ungulados (66.3\%) y las aves (17.7\%). Entre los ungulados, la especie más cazada es Tayassu pecari con 32 animales/año y entre las aves, la más cazada es Penelope jacquacu con 13 animales/año (Tabla 1). En términos de biomasa, fueron extraídos $2123.9 \mathrm{~kg} /$ año de las áreas de uso del parque y de 2678.2 kg/año de la zona de amortiguamiento. Siendo la especie que contribuyó con la mayor biomasa el Tayassu pecari con $937.6 \mathrm{~kg} /$ año en las áreas de uso del parque y $1230 \mathrm{~kg} /$ año en la zona de amortiguamiento (Tabla 1).

Del total de especies cazadas, seis (mamíferos) se tomaron en cuenta con el modelo de cosecha de Robinson y Redford (1991), las cuales son especies permitidas para la caza dentro de las áreas de uso del parque. En todos los casos la cuota de cosecha potencial sostenible es menor que la tasa de extracción real, lo que sugiere que la caza de estas especies es sustentable (Tabla 2).

En cuanto a la pesca, este manejo de fauna dio resultados positivos, en cuanto a la eliminación de tóxicos y explosivos como herramientas de uso. Los usuarios utilizaron como artes de pesca, anzuelos, tarrafas y redes de diámetros adecuados. Los peces mayormente extraídos fueron, carachamas, boquichicos, mojarras, añashuas, yulillas y bagres.

Los usuarios se registraron en el Puesto de Control, antes de ingresar a las áreas de uso del parque. De la misma forma a su salida proporcionaron información de su cosecha a los Guardaparques. Los facilitadotes fueron los responsables de registrar la información de cosecha de caza y pesca en sus respectivas comunidades. 
En otros sectores, las comunidades del sector Pikiyacu, utilizan el área de manejo, localizado en la zona de recuperación del parque, abarcando parte de la cuenca del río Pikiyacu y presenta una zona de uso (caza y pesca), teniendo esta una extensión de $10 \mathrm{~km} 2$.

El manejo comunal de fauna silvestre en este sector se desarrolla por las comunidades de Los Ángeles, Nuevo Trujillo y Puerto Franco. Además, se tiene avances significativos en los sectores de Chazuta (noroeste del parque), Pucayacu (suroeste del parque) y Cushabatay (noreste del parque). En Chazuta, se ubican las comunidades de Ramón Castilla, Santa Rosa de Chipaota, Canayo y Callanayacu. En el sector de Cushabatay se encuentran ubicadas las comunidades nativas de Libertad e Isolaya, así como las comunidades ribereñas de Nuevo Álan, Belaunde y Nuevo San Martín. Por último, en el sector Pucayacu se ubican las comunidades de Maronilla, Consuelo, Nuevo San Martín y Gossen.

\section{DISCUSIÓN}

La participación de los pobladores locales y el intercambio de información en el diseño e implementación de las acciones de manejo comunal de fauna, es muy importante para la adecuada consecución de los objetivos trazados.

En términos generales, los cazadores respetaron las normas de uso, esto se reflejó por ejemplo en la disminución de la cosecha en primates, el cual se produjo en dos oportunidades dentro del año de manejo, en comparación de años anteriores, donde la cacería en estas especies era intensiva y notoria.

Mediante las capacitaciones oportunas brindadas a las autoridades y pobladores locales, las actividades de manejo comunal de fauna se desarrollaron adecuadamente. Los pobladores locales siguen interesados y motivados en continuar con este proceso y seguir participando junto al equipo técnico y el Parque, en estas acciones de manejo.
El manejo comunal de fauna es una estrategia que no fue aplicada de la misma manera en las diferentes comunidades que participan actualmente, se consideró que cada comunidad y sector de trabajo, tenía condiciones únicas, las cuales se tuvieron en cuenta al momento de iniciar las actividades de diseño del plan de manejo comunal de fauna.

La inclusión de aspectos biológicos y productivos al programa, sumado al contexto socio-cultural de las comunidades, garantizarán la viabilidad de las actividades de manejo y se mantendrá una relación armoniosa entre los pobladores locales, los ecosistemas y la fauna silvestre.

Los registros de caza y pesca, permitieron recopilar cualitativa y cuantitativamente el nivel de uso y áreas de extracción de los animales de caza y pesca. Basados en esta información, la cual debe ser sistematizada y analiza constantemente, se puede tomar las medidas correctivas del caso, evitando sobre explotación de la fauna silvestre. Por otro lado, la falta de registros de animales cazados y la cacería furtiva aún presente en la zona, permiten sugerir que las poblaciones de las especies mencionadas (Tabla 2), se estarían cazando en mayor cantidad, por lo cual los datos mencionados serían subestimaciones.

El modelo de cosecha de Robinson y Redford (1991) es útil como una herramienta de primera mano para guiar las primeras acciones de manejo comunal en áreas donde es necesario combinar el uso y la conservación de la fauna silvestre. Las imposiciones estatales y externas, no funcionan, el proceso participativo desarrollado en este programa, establece resultados positivos y augura la continuidad de las actividades a largo plazo.

El manejo comunal de fauna silvestre debe ser un proceso continuo, que se debe extender en el tiempo y convertirse en una actividad permanente dentro de las comunidades. Se espera que este modelo de manejo, puesto en marcha inicialmente en 11 comunidades, pueda ser replicado en otras alrededor del parque. 


\section{AGRADECIMIENTOS}

Nuestra mayor consideración a las autoridades y pobladores de las comunidades del valle del Ponasa y Pikiyacu, por su dedicación, amabilidad y apoyo en este programa. A los pobladores de la comunidad de Canayo por la confianza depositada en el responsable del programa y por la grata experiencia adquirida durante el desarrollo de las acciones de manejo. Agradecemos de igual forma a los Guardaparques que colaboraron en las diferentes actividades del programa. Mi sincero reconocimiento a Miguel Vásquez, compañero e infatigable colaborador de campo.

\section{REFERENCIAS BIBLIOGRÁFICAS}

CENTRO DE CONSERVACIÓN, INVESTIGACIÓN Y MANEJO DE AREAS NATURALES (CIMA). 2004. Plan Maestro del Parque Nacional Cordillera Azul. Perú. Pp 6.

GAVIN, M. 2002. An Assessment of forest use value in the Northern Peruvian Amazon. Connecticut University. United State American.

NOVARO, A. J., K. H. REDFORD \& R. E. BODMER 2000. Effect of Hunting in Source-Sink Systems in the Neotropics. Conservation Biology 14: 713-721.

WALI, A., J. CHIRA \& H DEL CAMPO. 2003. Reporte final sobre el mapeo de usos y fortalezas del Parque Nacional Cordillera Azul. Perú. Pp 4.

WEBB, R. \& G. FERNÁNDEZ (2001). Perú en Número. Instituto Cuanto. Lima.

WATANABE, J. 2004. Actividades de Manejo Comunal de Fauna Silvestre en el Sector Noroeste del Parque Nacional Cordillera Azul. Centro de Conservación, Investigación y manejo de Areas (CIMA). Perú. Pp. 5.

WATANABE, J. 2005. Diagnóstico del Programa de Manejo Comunal de Fauna Silvestre en el Parque Nacional Cordillera Azul y Zona de Amortiguamiento. Perú. Pp. 2.

ZAPATA, J. 2001. Sustentabilidad de la Cacería de Subsistencia: El Caso de Cuatro Comunidades Quichuas en la Amazonía Nororiental Ecuatoriana. Journal Neotropical Mammal, 8 (1) 5966.

ROBINSON, J. \& H. REDFORD. 1991. Uso y Conservación de la Vida Silvestre Neotropical. Chicago. Pp. 485-501. 


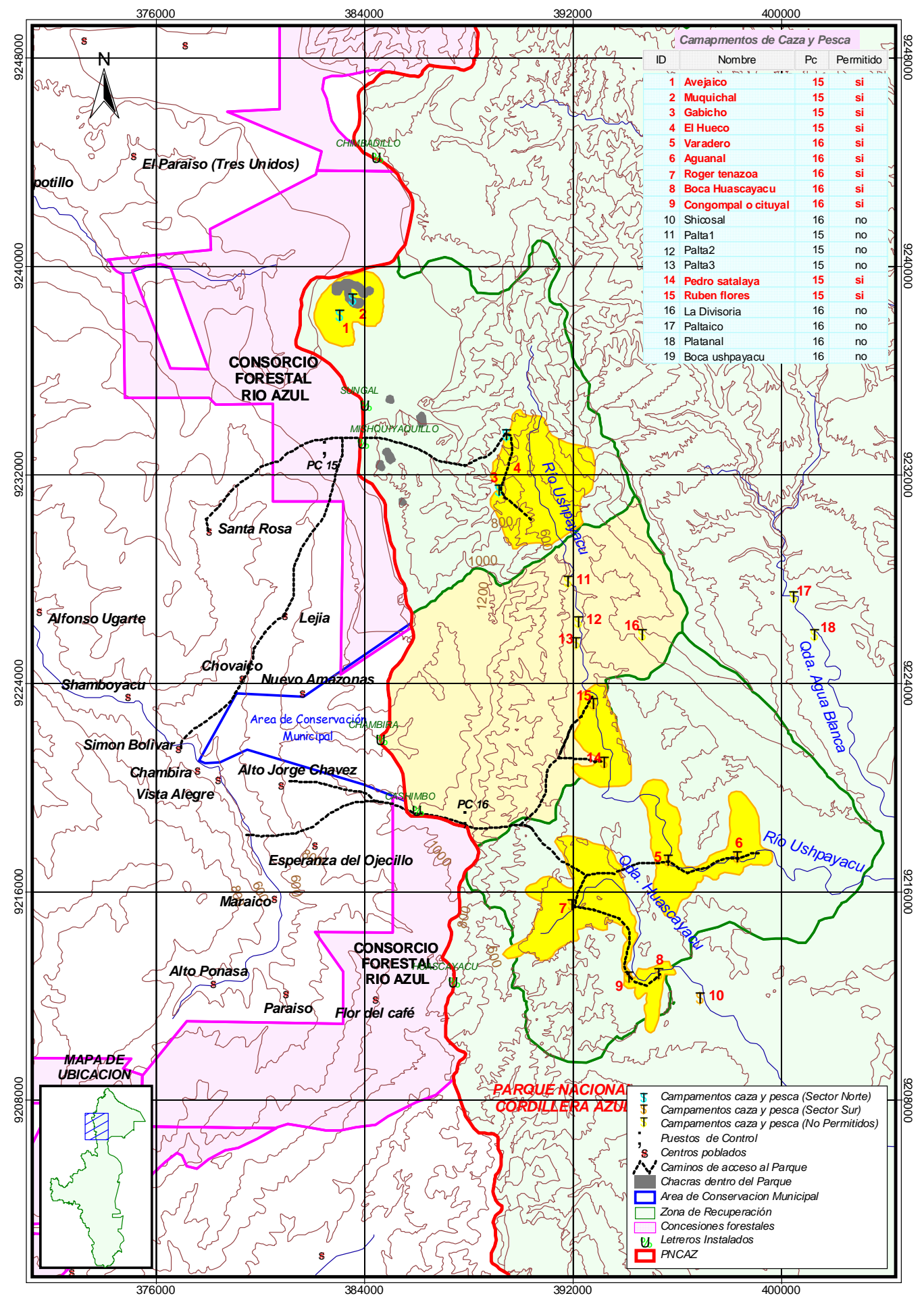

Figura 2: Mapa del área de recuperación del parque mostrando las areas de uso del sector Shamboyacu. 
Tabla 1. Especies cazadas en las áreas de uso del parque y en la zona de amortiguamiento, durante los meses de octubre 2004 a setiembre 2005 (Sector Shamboyacu).

\begin{tabular}{|c|c|c|c|c|c|}
\hline \multirow[t]{2}{*}{ Especies } & \multirow[t]{2}{*}{$\begin{array}{l}\text { Peso } \\
\text { (Kilos) }\end{array}$} & \multicolumn{2}{|c|}{$\begin{array}{c}\text { Cosecha Real } \\
\text { (Número de Individuos) }\end{array}$} & \multicolumn{2}{|c|}{$\begin{array}{c}\text { Biomasa } \\
(\mathrm{Kg})\end{array}$} \\
\hline & & Parque & $\mathrm{ZA}$ & Parque & $\mathrm{ZA}$ \\
\hline \multicolumn{6}{|l|}{ Ungulados } \\
\hline Tayassu tajacu & 19.7 & 25 & 29 & 492.5 & 571.3 \\
\hline Tayassu pecari & 29.3 & 32 & 42 & 937.6 & 1230.6 \\
\hline Mazama americana & 28 & 18 & 16 & 504.0 & 448.0 \\
\hline \multicolumn{6}{|l|}{ Primates } \\
\hline Ateles paniscus & 17.0 & 3 & & 51.0 & \\
\hline \multicolumn{6}{|l|}{ Roedores } \\
\hline Cuniculus paca & 8.1 & 3 & 40 & 24.3 & 48.1 \\
\hline Dasyprocta fuliginosa & 3.1 & 6 & 43 & 18.6 & 133.3 \\
\hline \multicolumn{6}{|l|}{ Desdentados } \\
\hline Dasypus novemcinctus & 5.0 & 5 & 22 & 25.0 & 110.0 \\
\hline \multicolumn{6}{|l|}{ Carnívoros } \\
\hline Nasua nasua & 3.9 & 1 & 1 & 3.9 & 3.9 \\
\hline Potos flavus & 2.5 & & 6 & & 15.0 \\
\hline Felis pardales & 10.5 & & 1 & & 10.5 \\
\hline \multicolumn{6}{|l|}{ Edentados } \\
\hline Myrmecophaga tridáctila & 22.0 & & 1 & & 22.0 \\
\hline \multicolumn{6}{|l|}{ Aves } \\
\hline Pipile cumanenses & 3.5 & 1 & & 3.5 & \\
\hline Mitu tuberosa & 5.0 & 2 & & 10.0 & \\
\hline Tinamus sp. & 2.0 & 4 & 6 & 8.0 & 12.0 \\
\hline Penelope jacquacu & 3.5 & 13 & 16 & 45.5 & 56.0 \\
\hline Ortalis guttata & 2.5 & & 1 & & 2.5 \\
\hline Ramphastos sp. & 3.0 & & 1 & & 3.0 \\
\hline \multicolumn{6}{|l|}{ Reptiles } \\
\hline Geochelone denticulada & 12.0 & & 1 & & 12.0 \\
\hline
\end{tabular}

Tabla 2. Cálculo de la cuota de extracción sustentable para los mamíferos permitidos en las áreas de uso del parque, con base en el modelo de Robinson y Redford (1991).

\begin{tabular}{|c|c|c|c|c|c|c|c|c|c|c|}
\hline \multirow[t]{3}{*}{ Especies } & \multirow{3}{*}{$\begin{array}{l}\text { Pmax } \\
(\text { Ind/km²) }\end{array}$} & \multirow{3}{*}{$\begin{array}{c}\text { PCS } \\
(\text { Ind/km²) }\end{array}$} & \multicolumn{5}{|c|}{ PCS en las Areas de Uso (Ind/ km2) } & \multicolumn{2}{|c|}{ Extracción } & \multirow[t]{3}{*}{$\mathrm{S}$} \\
\hline & & & A1 & $\mathrm{A} 2$ & A3 & A4 & A5 & Total & Real & \\
\hline & & & $15 \mathrm{~km}^{2}$ & $10 \mathrm{~km}^{2}$ & $14 \mathrm{~km}^{2}$ & $7 \mathrm{~km}^{2}$ & $5 \mathrm{~km}^{2}$ & $51 \mathrm{~km}^{2}$ & Ind/51 km² & \\
\hline \multicolumn{11}{|l|}{ Ungulados } \\
\hline Tayassu tajacu & 12.03 & 2.41 & 36 & 24 & 39 & 17 & 12 & 128 & 25 & SI \\
\hline Tayassu pecari & 4.12 & 0.83 & 13 & 8 & 12 & 6 & 4 & 43 & 32 & SI \\
\hline $\begin{array}{l}\text { Mazama americana } \\
\text { Roedores }\end{array}$ & 1.67 & 0.67 & 10 & 7 & 9 & 5 & 3 & 34 & 18 & SI \\
\hline Cuniculus paca & 6.56 & 1.31 & 20 & 13 & 18 & 9 & 7 & 67 & 3 & SI \\
\hline $\begin{array}{l}\text { Dasyprocta fuliginosa } \\
\text { Desdentados }\end{array}$ & 22.44 & 8.98 & 135 & 90 & 126 & 63 & 45 & 459 & 6 & SI \\
\hline Dasypus novemcinctus & 12.98 & 5.0 & 78 & 60 & 73 & 36 & 26 & 273 & 5 & SI \\
\hline
\end{tabular}

Pmax: Producción máxima (Robinson y Redford, 1991)

PCS: Potencial de Cosecha Sostenible (Robinson y Redford, 1991)

S: Sustentabilidad

A1: Area de uso Hueco-Gavicho
A2: Area de uso Varadero-Aguanal

A3: Area de uso Tenazoa-Huascayacu

A4: Area de uso Satalaya-Flores

A5: Area de uso Avejaico-Muquichal 